\title{
Efecto de la Dispersión de Radiación sobre el Rendimiento Cuántico en la Degradación Fotocatalítica de Ácido Dicloroacético
}

\author{
Jesús A. Mesa ${ }^{(1)}$, Fiderman Machuca-Martínez ${ }^{(1)}$, Miguel A. Mueses ${ }^{(1,2) *}$ \\ (1) Escuela de Ingeniería Química, Grupo de Investigación GAOX, Universidad del Valle, \\ A. A. 25360, Cali-Colombia \\ (2) Facultad de Ingeniería, Programa de Ingeniería Química, Universidad de Cartagena, \\ Av. Del Consulado, 48-152, Edf. Ingeniería, Cartagena-Colombia \\ (e-mail: mmueses@unicartagena.edu.co)
}

Recibido Jul. 29, 2011; Aceptado Sep. 30, 2011; Versión final recibida Nov. 20, 2011

\section{Resumen}

Se analizan diferentes modelos de dispersión de radiación para evaluar su efecto sobre la cantidad de energía radiante absorbida por un fotocatalizador en la determinación de rendimientos cuánticos. Se simuló el campo de radiación en un sistema de foto-descomposición heterogénea de ácido dicloroacético catalizada por partículas de dióxido de titanio utilizando un modelo unidimensional para evaluar la absorción y la dispersión de radiación en el espacio de reacción. Se compararon los resultados obtenidos con diferentes modelos de funciones de fase anisotrópicas respecto a la función de fase isotrópica. Se encontró que la dispersión de radiación isotrópica es una aproximación razonable para la dispersión producida por partículas en suspensión de dióxido de titanio.

Palabras clave: fotocatálisis, función de fase, dióxido de titanio, fotoreactores, modelado matemático

\section{Radiation Scattering Effect on Quantum Yield in the Photocatalytic Degradation of Dichloroacetic Acid}

\begin{abstract}
Different models of radiation scattering used to valuate its effect on the amount of radiant energy absorbed by a photocatalyst in the determination of quantum yields were analyzed. The radiation field in a heterogeneous photodecomposition of dichloroacetic acid catalyzed by particles of titanium dioxide was simulated using a one-dimensional model to evaluate the absorption and scattering of radiation in the reaction space. The results obtained by the different models of nonisotropic phase function with the isotropic phase function were compared. It was found that the scattering of isotropic radiation is a reasonable approximation for scattering produced by suspended particles of titanium dioxide.
\end{abstract}

Keywords: photocatalysis, phase function, titanium dioxide, photoreactors, mathematical modeling 


\section{INTRODUCCIÓN}

La fotocatálisis heterogénea es un proceso que se fundamenta en la absorción de energía radiante directa o indirecta por parte de un semiconductor, para generar especies transitorias tales como duplas electrón-hueco y radicales hidroxilo $\left(\mathrm{OH}^{*}\right)$. Estos procesos dependen fuertemente de la activación del semiconductor por lo cual es indispensable cuantificar la velocidad local de absorción de fotones (o LVRPA, por sus siglas en ingles) (Henderson, 2011).

Un modelo de cinética fotocatalítica heterogénea, depende de tres aspectos fundamentales: el mecanismo cinético de la reacción, el rendimiento cuántico del proceso (cantidad de moles transformadas por cantidad de energía absorbida en el sistema) y la velocidad de absorción de energía por parte del semiconductor (Ryu y Choi, 2008).

Los fenómenos ópticos de absorción y dispersión en procesos de reacción, generalmente son modelados considerando la dispersión de radiación como un fenómeno no aislado, que se puede describir de forma ideal con distribuciones isotrópicas de energía dentro del volumen de reacción. Esta consideración no permite, claramente, establecer los efectos sobre la velocidad de reacción y su relación con la velocidad de absorción de energía (Korkin et al., 2011; Maddigapu et al., 2011).

No existe un método estándar para medir experimentalmente la velocidad de absorción de energía en el fotocatalizador, sin embargo se pueden hacer aproximaciones para algunas geometrías sencillas y sistemas experimentales modelo (Paušová et al., 2011; Ismail y Bahnemann, 2011; Emeline et al., 2006) que permiten la solución de la ecuación de transferencia radiativa (RTE), a través del método de la Ordenada Discreta (DOM).

La importancia de evaluar los rendimientos cuánticos como parámetros comparativos se establece por la naturaleza de los procesos de inducción de duplas electrón $(\mathrm{e}-)$ / hueco $(\mathrm{h}+)$ en la interface semiconductor/ solución, donde la velocidad de reacción en la etapa primaria de excitación del catalizador es considerada fundamental en el proceso. El uso de rendimiento cuántico como parámetro de evaluación de catalizadores y reacciones fotocatalíticas es de amplio uso, tanto en mezclas como en sustancias modelo; en particular este parámetro es útil para el modelado, simulación y escalamiento de reactores. (Sawage et al., 2010; Braham y Harris, 2009). Normalmente, se trabaja con un sistema isotropico, el cual no tiene en cuenta el apantallamiento de la luz y sus interacciones con el catalizador y las sustancias reaccionantes (Lao, 1996).

El desarrollo de este trabajo se fundamenta en la selección y análisis de distintos modelos que describan el fenómeno de dispersión de radiación para evaluar su efecto sobre los parámetros que caracterizan la cantidad de energía radiante absorbida por el catalizador, particularmente el rendimiento cuántico primario del sistema.

\section{MODELO MATEMÁTICO}

El modelo utilizado se basa en la definición de rendimiento cuántico monocromático inicial, ecuación (1):

$\left.\phi_{\lambda}^{0}\right]_{T}=\frac{\left[\left(\left(R_{\text {Hom }, i}^{\text {Seudo }}\right)_{\lambda}^{t \rightarrow 0}\right\rangle_{V R}\right]_{\text {EXPERIM. }}}{\left[\left(e_{\lambda}^{a}\right\rangle_{V R}\right]_{\text {CALCULADA }}}$

Donde $R$ es la velocidad inicial de reacción seudo-homogénea del componente $\mathrm{i}$ y $e^{a}{ }_{\lambda}$ es la velocidad volumétrica local de absorción de fotones, ambas promediadas en todo el volumen de reacción $V_{R}$.

Para calcular la ecuación (1) se debe resolver la RTE en el sistema de reacción. Al considerar que la radiación difusa es parte del sistema, es posible calcular el campo radiante con un modelo 
unidimensional. La ecuación (2) es la RTE simplificada para el sistema de reacción, con las respectivas condiciones de frontera (3).

$$
\begin{aligned}
& \mu \frac{\partial i_{\lambda}^{\prime}(x, \mu)}{\partial x}+\left(\alpha_{\lambda}+\sigma_{S \lambda}\right) i_{\lambda}^{\prime}(x, \mu)-\alpha_{\lambda} i_{b \lambda}^{\prime}=\frac{\sigma_{S \lambda}}{4 \pi} \int_{4 \pi} i_{\lambda}^{\prime}(x, \mu) \Phi\left(\mu, \mu^{\prime}\right) d \mu^{\prime} \\
& i_{\lambda}^{\prime}(0, \mu)=i_{\lambda}^{\prime 0}, \quad \mu>0 \quad ; \quad i_{\lambda}^{\prime}\left(L_{R}, \mu\right)=0, \quad \mu<0
\end{aligned}
$$

Donde $\mu=\cos \theta, i_{\lambda}{ }_{\lambda}$ es la intensidad específica de radiación, $\alpha_{\lambda}$ es el coeficiente de absorción, $\sigma_{s \lambda}$ es el coeficiente de dispersión (coeficiente de scattering), $i_{\lambda}{ }^{0}{ }^{0}$ es el flujo del espectro de radiación que entra en el medio de reacción, y $\Phi\left(\mu, \mu^{\prime}\right)$ es la función de fase, que se puede interpretar como la razón de la intensidad que se dispersa en una dirección con la intensidad que se dispersa en esa dirección.

En este estudio, se utilizó como base de comparación de los modelos planteados por el grupo de Cassano y Alfano (Zalazar et al., 2005; Cassano et al., 2000; Alfano et al., 1995; Alfano et al., 1985) para un reactor de mezcla perfecta y la solución rigurosa de la RTE. Así, la ecuación (4) representa el comportamiento del sistema de reacción:

$$
\left\langle R_{\mathrm{Hom}, i}^{\text {Seudo }}(x, t)\right\rangle_{V_{R}}=\frac{V_{T}}{V_{R}}\left[\left[\frac{d C(t)}{d t}\right]_{T k}\right]_{\lambda}^{t \rightarrow 0}
$$

A partir de este balance, se considera que las ecuaciones para predecir los flujos radiativos directos e indirectos (reflejados) del sistema parabólico de emisión (reflector y lámpara) son:

$$
\begin{aligned}
& q_{D, \lambda}\left(r_{1}, \beta_{1}\right)=K_{L, V} \int_{\phi_{D, 1}}^{\phi_{D, 2}} \Delta \rho^{\prime}(\phi)\left[\cos \theta_{D, 2}(\phi)-\cos \theta_{D, 1}(\phi)\right] \sin \phi d \phi \\
& q_{I n, \lambda}\left(r_{1}, \beta_{1}\right)=K_{L, V} \Gamma_{R f, \lambda} \int_{\phi_{I n 1}}^{\phi_{I n, 2}} \Delta \rho_{E}^{\prime}(\phi)\left[\cos \theta_{I n, 2}(\phi)-\cos \theta_{I n, 1}(\phi)\right] \sin \phi d \phi
\end{aligned}
$$

En las ecuaciones (5) y (6) $K_{L, V}$ es la constante característica de la lámpara que puede obtenerse a partir del tipo de emisión (volumétrica o superficial) y la potencia espectral de salida de la lámpara $\left(E_{\lambda}\right)$. Así, el flujo radiativo total está dado por:

$$
\begin{aligned}
& q_{T, \lambda}\left(r_{1}, \beta_{1}\right)=q_{D, \lambda}\left(r_{1}, \beta_{1}\right)+q_{I n, \lambda}\left(r_{1}, \beta_{1}\right) \\
& \left\langle q_{T, \lambda}\left(r_{1}, \beta_{1}\right)\right\rangle_{A p}=\left(\frac{4}{\pi r_{P}^{2}}\right) \int_{0}^{r_{P}} r_{1} d r_{1} \int_{0}^{\frac{\pi}{2}} q_{T, \lambda}\left(r_{1}, \beta_{1}\right) d \beta
\end{aligned}
$$

A partir del flujo de radiación y el coeficiente de transmisión espectral $Y_{P, \lambda}$, se puede obtener el flujo del espectro de radiación:

$$
i_{\lambda}^{\prime 0}=\frac{1}{\pi} Y_{P, \lambda} \cdot\left\langle q_{T, \lambda}\left(r_{1}, \beta_{1}\right)\right\rangle_{A_{p}}
$$

Por lo tanto, la velocidad volumétrica local de absorción de fotones (LVRPA) puede ser calculada mediante la siguiente expresión:

$$
e^{a}(x)=\int_{\lambda \min }^{\lambda \max } \alpha_{\lambda} i_{\lambda}^{\prime}(x, \mu) d \mu d \lambda
$$

donde $\lambda_{\min }$ y $\lambda_{\max }$ es el intervalo útil de absorción para el TiO2 (300 - $450 \mathrm{~nm}$ respectivamente). 
Dado que el campo de radiación es no uniforme, se debe calcular el promedio en el volumen de reacción. La ecuación (11) es la adecuada para el modelo unidimensional, si la sección transversal del reactor es constante y la distribución del catalizador es uniforme:

$$
\left\langle e_{\lambda}^{a}\right\rangle_{V_{R}}=\frac{1}{L_{R}} \int_{0}^{L_{R}} e_{\lambda}^{a}(x) d x
$$

Al resolver las ecuaciones (1), (4) y (11) se obtiene el rendimiento cuántico para el reactor:

$$
\left.\phi_{\lambda}^{0}\right]_{T}=\frac{\left\{\frac{V_{T}}{V_{R}}\left[\left[\frac{d c(t)}{d t}\right]_{T k}\right]_{\lambda}^{t \rightarrow 0}\right\}_{E X P .}}{\left\{\frac{1}{L_{R}} \int_{0}^{L_{R}} e_{\lambda}^{a}(x) d x\right\}_{C A L C .}}
$$

Finalmente, para la validación de los modelos se consideró los datos experimentales reportados en la literatura (Zalazar et al., 2005; Brandi et al., 2001; Alfano et al., 1995; Cabrera et al., 1996).

\section{SOLUCIÓN DEL MODELO}

La ecuación integro-diferencial RTE se transforma por el método DOM en un sistema de ecuaciones diferenciales ordinarias, donde se obtienen dos sistemas N/2 de ecuaciones de ordenada discreta, donde $N$ es el orden de aproximación.

$$
\begin{aligned}
& {\left[\begin{array}{c}
\mu_{1} i_{1}^{\prime+} \\
\mu_{2} i_{2}^{++} \\
\vdots \\
\mu_{n} i_{n}^{\prime+}
\end{array}\right]=\left[\begin{array}{c}
1 \\
1 \\
\vdots \\
1
\end{array}\right] \frac{\alpha_{\lambda}}{K_{\lambda}} i_{b \lambda}^{\prime}+} \\
& \left.\frac{\sigma_{S \lambda}}{4 \pi K_{\lambda}}\left\{\begin{array}{cccc}
\Phi\left(\mu_{1} \mu_{1}\right) & \Phi\left(\mu_{1} \mu_{2}\right) & \cdots & \Phi\left(\mu_{1} \mu_{n}\right) \\
\Phi\left(\mu_{2} \mu_{1}\right) & \Phi\left(\mu_{2} \mu_{2}\right) & \cdots & \Phi\left(\mu_{2} \mu_{n}\right) \\
\vdots & \vdots & \ddots & \vdots \\
\Phi\left(\mu_{n} \mu_{1}\right) & \Phi\left(\mu_{n} \mu_{2}\right) & \cdots & \Phi\left(\mu_{n} \mu_{n}\right)
\end{array}\right]\left[\begin{array}{cccc}
w_{1} & 0 & \cdots & 0 \\
0 & w_{2} & \cdots & 0 \\
\vdots & \vdots & \ddots & \vdots \\
0 & 0 & \cdots & w_{n}
\end{array}\right]-\frac{4 \pi K_{\lambda}}{\sigma_{S \lambda}} I\right\} i_{\lambda}^{\prime+} \\
& {\left[\begin{array}{c}
\mu_{1} i_{1}^{\prime^{+}} \\
\mu_{2} i_{2}^{\prime+} \\
\vdots \\
\mu_{n} i_{n}^{\prime+}
\end{array}\right]=\left[\begin{array}{c}
1 \\
1 \\
\vdots \\
1
\end{array}\right] \frac{\alpha_{\lambda}}{K_{\lambda}} i_{b \lambda}^{\prime}+} \\
& \frac{\sigma_{S \lambda}}{4 \pi K_{\lambda}}\left\{\left[\begin{array}{cccc}
\Phi\left(-\mu_{1} \mu_{1}\right) & \Phi\left(-\mu_{1} \mu_{2}\right) & \cdots & \Phi\left(-\mu_{1} \mu_{n}\right) \\
\Phi\left(-\mu_{2} \mu_{1}\right) & \Phi\left(-\mu_{2} \mu_{2}\right) & \cdots & \Phi\left(-\mu_{2} \mu_{n}\right) \\
\vdots & \vdots & \ddots & \vdots \\
\Phi\left(-\mu_{n} \mu_{1}\right) & \Phi\left(-\mu_{n} \mu_{2}\right) & \cdots & \Phi\left(-\mu_{n} \mu_{n}\right)
\end{array}\right]\left[\begin{array}{cccc}
w_{1} & 0 & \cdots & 0 \\
0 & w_{2} & \cdots & 0 \\
\vdots & \vdots & \ddots & \vdots \\
0 & 0 & \cdots & w_{n}
\end{array}\right]-\frac{4 \pi K_{\lambda}}{\sigma_{S \lambda}} I\right\} i_{\lambda}^{\prime-}
\end{aligned}
$$


Con las condiciones de frontera:

$$
\begin{aligned}
& i_{\lambda}^{\prime+}(0)=i_{\lambda}^{\prime 0} \\
& i_{\lambda}^{\prime-}(L)=0
\end{aligned}
$$

La condición de frontera en $x=0,\left(i_{\lambda}^{\prime}{ }^{0}\right)$ se halla por medio de la ecuación (9) donde las integrales de las ecuaciones (7) y (8) se hallan por el método de Gauss-Kronrod.

Debido a los valores típicos de las propiedades ópticas no direccionales del sistema se hace necesario el uso de un algoritmo de solución con autocontrol del error, donde la modificación realizada consiste en medir el error con respecto a la norma euclidiana de la diferencia de los vectores solución de quinto orden con respecto a la solución de cuarto orden y no cambiar el tamaño local de paso sino la tolerancia inicial con el fin de obtener vectores dimensionales igualmente espaciados. Esta modificación se acoplo al método adaptativo modificado de RungeKutta-Fehlberg.

Se debe considerar la solución trivial del sistema cuando se considera que la emisión del medio es despreciable $\left(i_{b \lambda}^{\prime}=0\right)$, ya que la teoría de matriz fundamental garantiza que esta sea la única solución posible (ecuaciones 14 a 16). Por lo tanto, encontrar la solución del modelo se limita a encontrar los valores de $i^{i^{+}}{ }_{\lambda}$.

Por último se calculan relaciones de rendimientos cuánticos para diferentes funciones de fase respecto a la función de fase isotrópica mediante la siguiente expresión:

$$
\frac{\Phi^{*}}{\Phi_{\text {isotrópico }}}=\frac{\left[\int_{0}^{L_{R}} e_{\lambda}^{a}(x) d x\right]_{\text {isotrópico }}}{\left[\int_{0}^{L_{R}} e_{\lambda}^{a}(x) d x\right]^{*}}
$$

\section{RESULTADOS Y DISCUSIÓN}

El transporte de radiación policromática en un medio participativo y reactivo (lo cual implica absorción, dispersión y reacción química) es de suma complejidad, que sólo tiene solución analítica para situaciones muy simplificadas. Los modelos bidimensionales - bidireccionales no pueden resolverse analíticamente. Pueden utilizarse diferentes aproximaciones tanto analíticas como numéricas (dos Santos et al., 2011; Mueses y Machuca, 2010). El Método de la Ordenada Discreta (DOM) que se desarrolló dentro del marco de la teoría generalizada de transporte, es el procedimiento numérico más poderoso para la solución de los problemas de transferencia radiativa. Los modelos fueron resueltos por el método de la ordenada discreta de orden 8.

\section{Dispersión para partículas con reflexión especular}

En la Figura 1 se muestra el comportamiento de la LVPRA para la función de fase isotrópica comparado con la función de fase con reflexión especular para soluciones de $\mathrm{TiO}_{2}$ con concentraciones de $0.5 \mathrm{~g} / \mathrm{L}$ y de $1 \mathrm{~g} / \mathrm{L}$. Como se evidencia en la figura a medida que aumenta la concentración de catalizador la diferencia entre los dos modelos se incrementa, esto debido a que a mayores concentraciones de catalizador la dispersión se vuelve más significativa. 


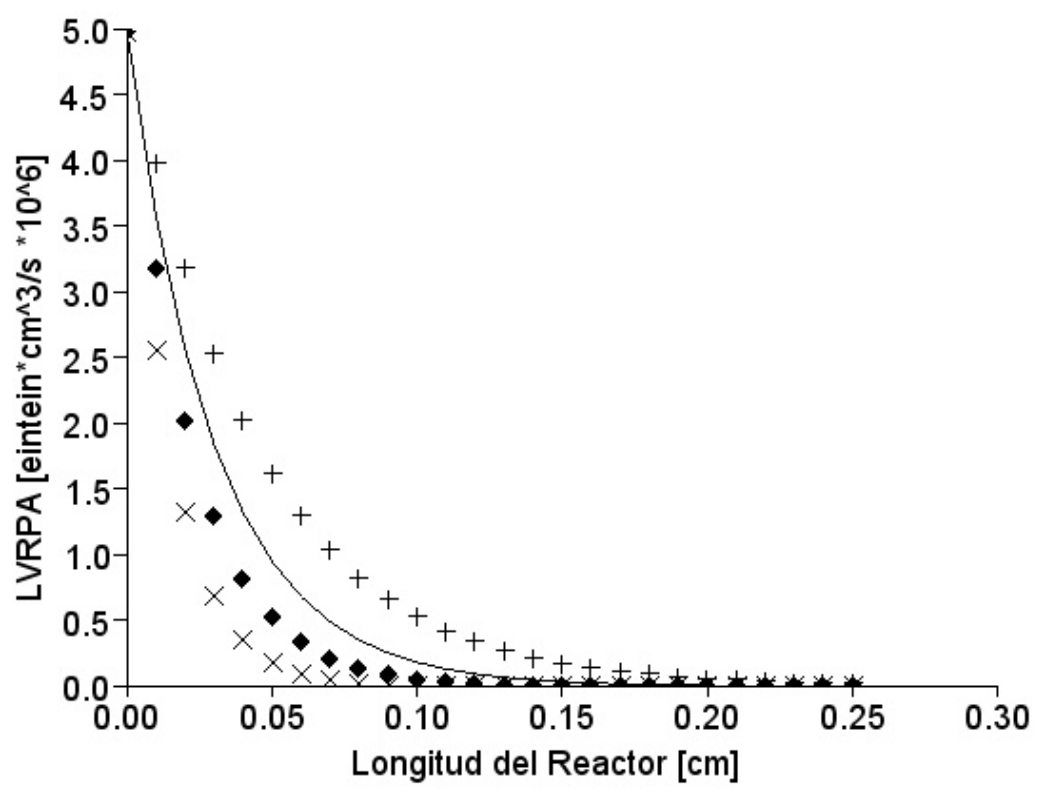

Fig. 1: LVPRA según los modelos de fase isotrópica y reflexión especular. (+) reflexión especular [1 g/l

$\mathrm{TiO}_{2}$ ], $(-)$ isotrópico [1 g/l TiO 2$],(\bullet)$ reflexión especular [0.5 g/l TiO 2 ], (x) isotrópico [0.5 g/l TiO 2$]$.

Según las leyes de la óptica geométrica, en los sistemas que poseen un comportamiento con reflexión especular, los rayos tienen mayor probabilidad de moverse hacia adelante (en la dispersión isotrópica, la dispersión puede ser en cualquier dirección) resultado que se evidencia en la Figura 1, debido a que los valores de la LVRPA para la reflexión especular siempre son mayores a los de la dispersión isotrópica. Esto último debido a que los rayos que se dispersan lo hacen hacia adelante, reincorporándose a la dirección de incidencia de radiación inicial, amortiguando el cambio brusco de energía radiante a lo largo del reactor.

Para el sistema en estudio, este comportamiento puede explicarse debido a que la dispersión para partículas con reflexión especular es válida para relaciones $\pi D / \lambda>5$ (donde $D$ es el diámetro de partículas y $\lambda$ es la longitud de onda), para el caso del $\mathrm{TiO}_{2}$ en el intervalo de trabajo se obtuvo que $\pi D / \lambda<\pi$, donde se puede concluir que el modelo es inapropiado para el sistema en cuestión.

\section{DISPERSIÓN DE RAYLEIGH}

Inicialmente se podría afirmar que el modelo es completamente inapropiado para el sistema partiendo de la fuerte restricción que implica el uso del modelo de Rayleigh $(D<<\lambda o \pi D / \lambda<0.6)$. En la Figura 2 se muestra el comportamiento de la LVRPA para el modelo de dispersión de Rayleigh a una concentración de $\mathrm{TiO}_{2}$ de 0.5 y 1g/L. La relación de rendimientos cuánticos $\Phi_{\text {Rayleigh }} / \Phi_{\text {isotrópico }}=0.416$, es decir, el sistema es $58 \%$ menos eficiente comparado respecto al comportamiento isotrópico debido a que según el modelo de Rayleigh, casi toda la energía es dispersada por las partículas. 


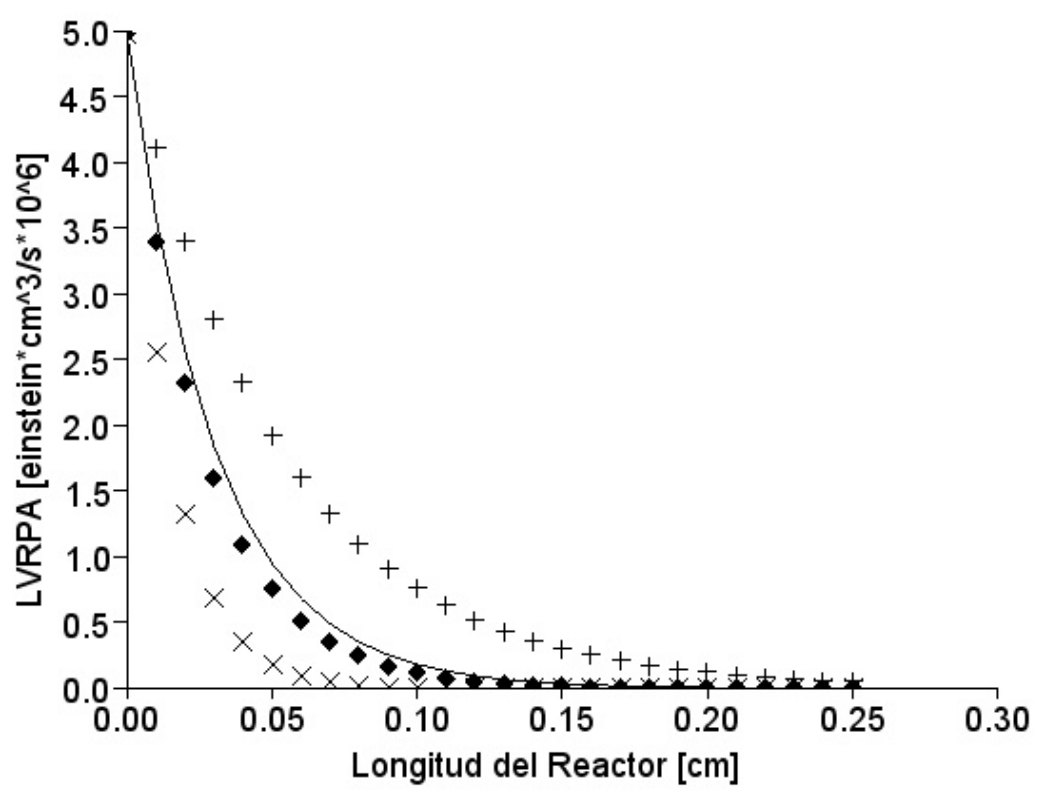

Fig. 2: LVPRA según los modelos de fase isotrópica y Rayleigh. (+) Rayleigh [1 g/l TiO $]$ ], ( -$)$ isotrópico [1

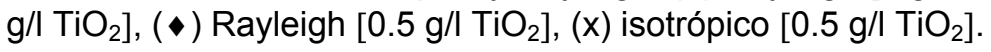

\section{DISPERSIÓN PARA PARTÍCULAS CON REFLEXIÓN DIFUSA}

La Figura 3 muestra el comportamiento de la LVRPA para la función de fase isotrópica comparado con la función de fase reflexión difusa a una concentración de $\mathrm{TiO}_{2}$ de 0.5 y $1 \mathrm{~g} / \mathrm{L}$.

La relación de rendimientos cuánticos $\Phi_{\text {difusd }} \Phi_{\text {isotrópico }}=1.166$ a una concentración de catalizador de $1 \mathrm{~g} / \mathrm{L}$, corresponde a un rendimiento $17 \%$ mayor con respecto al comportamiento isotrópico (el sistema necesita menos fotones absorbidos por unidad de tiempo para la misma degradación) esto debido a que las partículas con reflexión difusa generan una dispersión donde los rayos tienen mayor probabilidad de moverse hacia atrás y cierta probabilidad de moverse en cualquier dirección, lo que aumenta la probabilidad de absorción del medio. Esta probabilidad explica porque los valores de la LVRPA con reflexión difusa son siempre menores que los generados por la dispersión isotrópica.

\section{DISPERSIÓN DE MIE}

Este modelo se utiliza cuando las partículas están en el intervalo aproximado de $0.2 / \eta<D / \lambda<1$ en donde $\lambda$ la longitud de onda de la radiación incidente y $\eta$ el índice de refracción de las partículas. Para soluciones de $\mathrm{TiO}_{2}$, esta relación está dada entre $0.08<0.9<1$, lo que permite el uso del modelo de Mie.

Entonces, es de esperar que los resultados proporcionados por el modelo de Mie sean los mas aproximados. En la Figura 4 se muestra el comportamiento de la LVRPA según la dispersión de Mie, donde se evidencia claramente que los resultados son similares a los de la LVRPA con dispersión isotrópica $\left(\Phi_{\text {Mie }} / \Phi_{\text {isotrópico }}=1.021\right)$. 


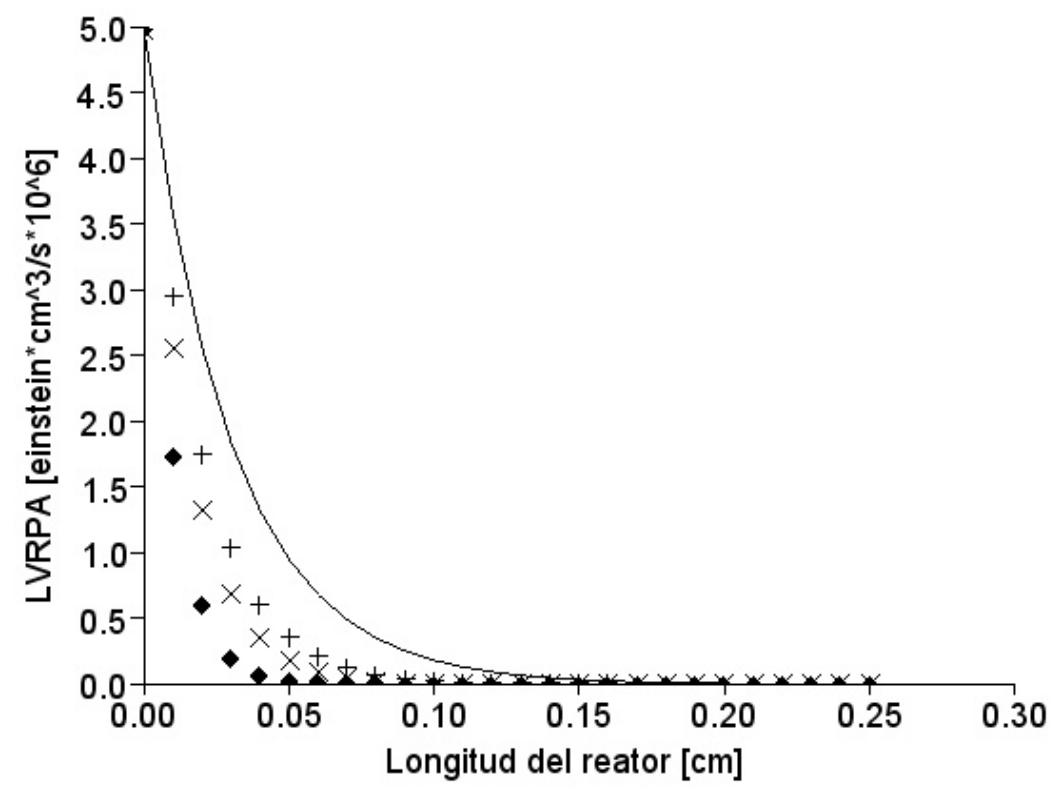

Fig. 3: LVPRA según los modelos de fase isotrópica y reflexión difusa. (+) reflexión difusa $\left[1 \mathrm{~g} / / \mathrm{TiO}_{2}\right],(-)$ isotrópico [1 $\left.\mathrm{g} / / \mathrm{TiO}_{2}\right],(\bullet)$ reflexión difusa $\left[0.5 \mathrm{~g} / \mathrm{l} \mathrm{TiO}{ }_{2}\right],(\mathrm{x})$ isotrópico $\left[0.5 \mathrm{~g} / \mathrm{l} \mathrm{TiO}{ }_{2}\right]$.

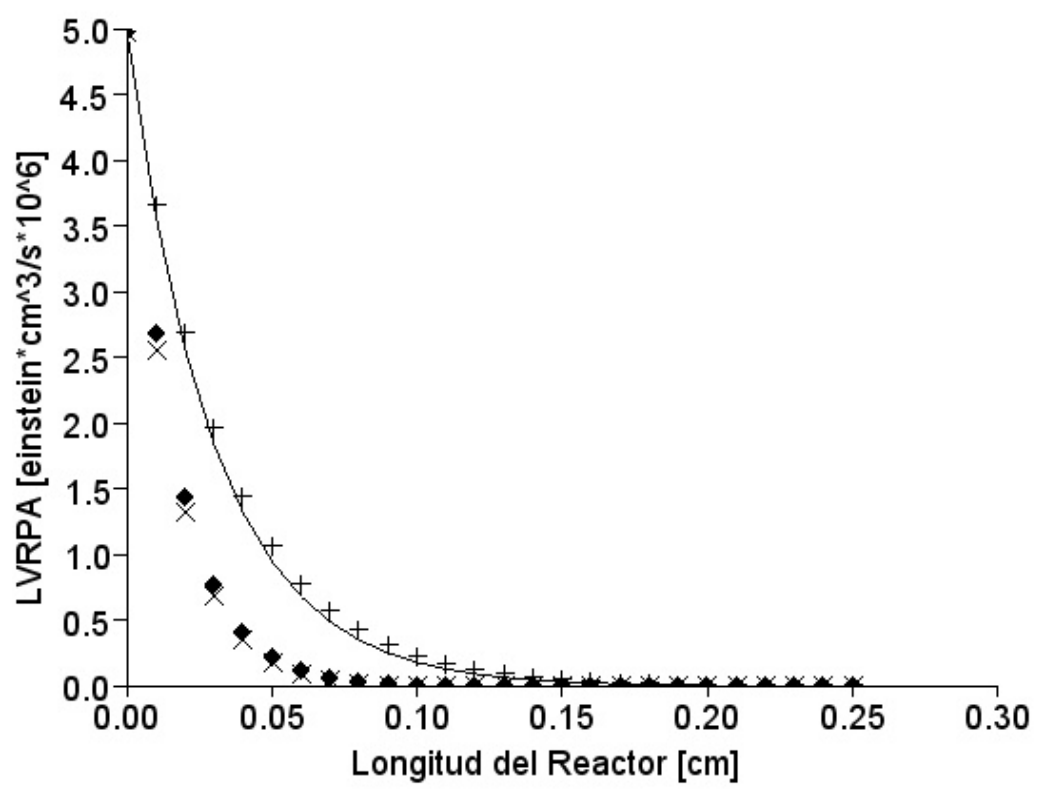

Fig. 4: LVPRA según los modelos de fase isotrópica y Mie. (+) Mie [1 g/l TiO $\left.{ }_{2}\right],(-)$ isotrópico $\left[1 \mathrm{~g} / / \mathrm{TiO}_{2}\right]$, $(\bullet)$ Mie $\left[0.5 \mathrm{~g} / / \mathrm{TiO}_{2}\right],(\mathrm{x})$ isotrópico $\left[0.5 \mathrm{~g} / \mathrm{l} \mathrm{TiO}{ }_{2}\right]$.

Estos resultados están en concordancia con lo reportado por Brandi et al., (2001), en este trabajo se encontró experimentalmente que la función de fase isotrópica es una aproximación razonable para la dispersión producida por las suspensiones de dióxido de titanio. En este trabajo la diferencia entre rendimientos cuánticos es sólo del $2 \%$.

Finalmente, la Tabla 1 resume los valores de la relación de rendimientos cuánticos para diferentes funciones de fase para soluciones de $\mathrm{TiO}_{2}$ a 0.5 y $1 \mathrm{~g} / \mathrm{L}$ con respecto a la función de fase isotrópica. Como puede observarse los modelos más adecuados son reflexión difusa y Mie,

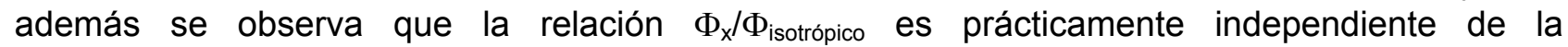
concentración, en el intervalo estudiado. 
Tabla 1: Relación $\Phi_{x} / \Phi_{\text {isotrópico }}$ para diferentes tipos de dispersión

\begin{tabular}{|c|c|c|c|c|c|}
\hline MODELO & Expresión matemática $\Phi(\varphi)$ & $\begin{array}{c}\Phi_{\mathrm{x}} / \Phi_{\text {isotrópico }} \\
{\left[1 \mathrm{~g} / \mathrm{T} \mathrm{TiO_{2 }}\right]}\end{array}$ & $\begin{array}{c}\Phi_{\mathrm{x}} / \Phi_{\text {isotrópico }} \\
{\left[0.5 \mathrm{~g} / \mathrm{l} \mathrm{TiO_{2 }}\right]}\end{array}$ & $\begin{array}{c}\Phi_{\mathrm{x}} / \Phi_{\text {Mie }} \\
{\left[1 \mathrm{~g} / \mathrm{TiO} \mathrm{Ti}_{2}\right]}\end{array}$ & $\begin{array}{c}\Phi_{\mathrm{x}} / \Phi_{\text {Mie }} \\
{\left[0.5 \mathrm{~g} / \mathrm{TTO} \mathrm{T}_{2}\right]}\end{array}$ \\
\hline $\begin{array}{c}\text { Reflexión } \\
\text { especular }\end{array}$ & $0.5 \rho_{\lambda}^{\prime}(\pi-\varphi) / \rho_{\lambda}$ & 0.679 & 0.702 & 0.665 & 0.677 \\
\hline $\begin{array}{c}\text { Reflexión } \\
\text { difusa }\end{array}$ & $8(\operatorname{sen} \varphi-\varphi \mu) / 3 \pi$ & 1.166 & 1.185 & 1.142 & 1.143 \\
\hline Rayleigh & $0.75\left(1+\mu^{2}\right)$ & 0.416 & 0.422 & 0.407 & 0.407 \\
\hline Mie & $0.6\left[(1-0.5 \mu)^{2}+(\mu-0.5)^{2}\right]$ & 1.021 & 1.037 & 1.000 & 1.000 \\
\hline
\end{tabular}

\section{CONCLUSIONES}

Se encontró que las relaciones de rendimientos cuánticos son prácticamente independientes de la concentración $\left(\Phi / \Phi_{\text {isotrópico }}\right)$ para cada tipo de dispersión.

Los modelos más adecuados para describir el comportamiento del sistema de reacción con $\mathrm{TiO}_{2}$ como catalizador son: la dispersión para partículas con reflexión difusa y el modelo de dispersión de Mie.

Se verifico, teóricamente, que la función de fase isotrópica es una aproximación razonable para la dispersión producida por las suspensiones de dióxido de titanio.

\section{AGRADECIMIENTOS}

Los autores agradecen a la Universidad del Valle (Colombia) con el proyecto 2520 "Modelamiento y Simulación de la Cinética Intrínseca de Reacciones Fotocatalíticas Heterogéneas" por la financiación del proyecto. Mueses y Machuca agradecen al Programa de Doctorados Nacionales de COLCIENCIAS, por la financiación de sus estudios doctorales.

\section{REFERENCIAS}

Alfano, O., R. Romero y A. Cassano, A Cilindrical Photoreactor Irradiated from de Bottom. I. Radiation Flux Density Generated by a Tubular Source and a Parabolic Reflector, Chemical Engineering Science: 40, 2119-2127 (1985).

Alfano, O. y otros tres autores, Scattering Effects Produced by Inert Particles in Photochemical Reactors. 1. Model and Experimental Verification, Industrial \& Engineering Chemistry Research: 34, 488-499 (1995).

Braham, R.J., y A.T.Harris, Review of Major Design and Scale-up Considerations for Solar Photocatalytic Reactors, Industrial \& Engineering Chemistry Research: 48(19), 8890-8905 (2009).

Brandi, R., O. Alfano y A. Cassano, Evaluation of Radiation Absorption in Slurry Photocatalytic Reactors. 1. Assessment of Methods in Use and New Proposal, Environmental Science \& Technology: 34(12), 2623-2630 (2001).

Cabrera, M., O. Alfano y A. Cassano, Absorption and Scattering Coefficients of Titanium Dioxide Particulate Suspensions in Water, Journal of Physical and Chemistry: 100, 20043-20050 (1996). 
Cassano, A. y O. Alfano, Reaction Engineering of Suspended Solid Heterogeneous Photocatalytic Reactors, Catalysis Today: 58(2-3), 167-197 (2000).

Dos Santos, E., C. Xavier y A. Petry, Estudio Numérico de Flujos Turbulentos Isotérmicos en Canales y Flujos Laminares con Convección Mixta en Cavidades, Información Tecnologica: 22(1), 71-82. (2011)

Emeline, A. y otros cuatro autores, Application of a Black Body Like Reactor for Measurements of Quantum Yields of Photochemical Reactions in Heterogeneous Systems, Journal of Physical Chemistry B: 110(14), 7409-7413 (2006).

Henderson, M., A Surface Science Perspective on TiO2 Photocatalysis, Surface Science Reports: 66 (6-7), 185-297 (2011).

Korkin, S.V., A.I. Lyapustin, y V.V. Rozanov, Analysis of the Radiative Transfer Equation with Highly Assymetric Phase Function, Journal of Quantitative Spectroscopy and Radiative Transfer: 112(10), 1595-1608 (2011).

Ismail, A. and D. Bahnemann, Mesostructured $\mathrm{Pt}_{\mathrm{TiO}} \mathrm{O}_{2}$ Nanocomposites as Highly Active Photocatalysts for the Photooxidation of Dichloroacetic Acid, Journal of Physical Chemistry C: 115(13), 5784-5791 (2011).

Lao, K., Inverted Regions in the Curve of Photodestruction Quantum Yield versus Photon Energy, Journal of Physical Chemistry: 100(12), 4693-4696 (1996).

Maddigapu, P., y otros cuatro autores, Modeling Phototransformation Reactions in Surface Water Bodies: 2,4-Dichloro-6-Nitrophenol as a Case Study, Environmental Science \& Technology: 45(1), 209-214 (2011).

Mueses, M. and F. Machuca, Una Solución de la Ecuación de Rachford-Rice para Sistemas Multifases Aplicando el Método Newton-Raphson, un Parámetro de Broyden y el Flash Negativo, Información Tecnológica: 21(4), 3-10 (2010).

Paušová, S. y otros cinco autores, Photocatalytic Properties of Aqueous Systems Containing $\mathrm{TiO}_{2}$ Nanoparticles, Catalysis Today: 161(1), 140-146 (2011).

Ryu, J. y W. Choi, Substrate-Specific Photocatalytic Activities of TiO2 and Multiactivity Test for Water Treatment Application, Environmental Science \& Technology: 42(1), 294-300 (2008).

Sagawe, G., y otros siete autores, Analysis of Photocatalytic Reactors Employing the Photonic Efficiency and the Removal Efficiency Parameters: Degradation of Radiation Absorbing and Nonabsorbing Pollutants, Industrial \& Engineering Chemistry Research: 49(15), 6898-6908, (2010).

Zalazar, C., y otros tres autores, Photocatalytic Intrinsic Reaction Kinetics I: Mineralization of Dichloroacetic Acid, Chemical Engineering Science: 60, 5240-5254 (2005). 